\title{
Fluid responsiveness in liver surgery: comparisons of different indices and approaches
}

\author{
Manuela Ferrario ${ }^{1 *}$, Salvatore Pala ${ }^{1}$, Federico Aletti ${ }^{1}$, Nicola Toschi ${ }^{2}$, Antonio Canichella ${ }^{2}$, Maria Guerrisi ${ }^{2}$, \\ Filadelfo Coniglione ${ }^{3}$, Giuseppe Baselli ${ }^{1}$ and Mario Dauri ${ }^{3}$
}

\author{
* Correspondence: \\ manuela.ferrario@polimi.it \\ ${ }^{1}$ Department of Electronics, \\ Information and Bioengineering \\ (DEIB), Politecnico di Milano, Piazza \\ Leonardo da Vinci 32, Milan 20133, \\ Italy \\ Full list of author information is \\ available at the end of the article
}

\begin{abstract}
The expected response to fluid infusion is an increase of cardiac output (CO), and this response depends mostly on the current cardiac function of the patient. The importance of the prediction of fluid responsiveness (FR) is based on the fact that fluid loading in hemodynamic unstable patients may be hazardous and dangerous, e.g., by exposing them to the risk of developing pulmonary edema. The objective of this work is to improve the knowledge about the performance of the indices of FR prediction in association with different classification approaches in a particular setting, i.e., liver surgery. The specific aims are (1) the comparison of different CO estimators from invasive arterial blood pressure (ABP) measurement with particular attention to the assessment of CO variation after fluid administration and (2) the comparison of several indices for the prediction of FR to maneuvers classified from the CO measurements provided by a commercial monitor (PiCCO ${ }^{\mathrm{TM}}$, Pulsion Medical System, Munich, Germany). The main finding of this work is that pulse pressure variation (PPV) indices are more reliable and computationally feasible than stroke volume variation (SW) indices. The PPV provided by PiCCO has the best performance in terms of area under curve, sensitivity, and specificity $(0.92,0.88$, and 0.86 , respectively), when the maneuvers are classified according to the maximum values of $\mathrm{CO}$ variation estimated during the second and third minutes after infusion. Moreover, $P P V_{\text {Picco }}$ is significantly correlated with the CO variation after infusion ( $r h o=0.51, p$ value $<0.05$ ). The threshold values produced by the PPV indices (PPV $=13.9 \%$ and PPV $\left._{\text {Picco }}=14.4 \%\right)$ are in agreement with the literature. From these observations, we conclude that the PPV index can be considered most suitable for the prediction of FR in liver surgery.
\end{abstract}

Keywords: Liver surgery; Fluid responsiveness; Pulse pressure variation; Stroke volume variation

\section{Background}

Hemodynamic monitoring plays an important role in the management of patients in intensive care and during major surgery. Functional hemodynamic monitoring can be defined as the assessment of the dynamic interactions of hemodynamic variables in response to a defined perturbation [1]. Clinical trials have shown the usefulness of functional hemodynamic monitoring for predicting volume responsiveness and identifying hidden cardiovascular insufficiency [2]. Fluids are primarily administered to reverse hypovolemia and hypotension. Hypovolemia may be due to external fluid losses caused by bleeding or losses from the gastrointestinal or urinary tracts, or internal

\section{Springer}

(c) 2014 Ferrario et al.; licensee Springer. This is an open access article distributed under the terms of the Creative Commons Attribution License (http://creativecommons.org/licenses/by/2.0), which permits unrestricted use, distribution, and reproduction in any medium, provided the original work is properly cited. 
losses due to extravasation of blood or exudation of body fluids. Optimal fluid resuscitation remains a matter of lively debate; for instance, there is no consensus about which intravenous fluid solution is ideal, and no secure data support a preference for one over another [3]. The end point of fluid resuscitation also remains unclear [3].

In the context of fluid management, the routine administration of fluids to ensure patient hydration must be separated from the maneuver called fluid challenge (FC), which refers to the initial volume expansion period performed to carefully evaluate the response of the patient to fluid infusion [4]. In particular, the importance of the prediction of fluid responsiveness (FR) relies on the fact that the fluid loading in such condition may be hazardous and dangerous for the patient, e.g., by causing or worsening pulmonary edema. Moreover, the fluid management of cirrhotic patients undergoing orthotopic liver transplantation (OLT) may be challenging and still remains controversial [5].

The expected response to fluid infusion is an increase of cardiac output (CO) or stroke volume (SV), and this response depends mostly on the position of an individual patient on the cardiac function curve, i.e., the Frank-Starling curve [6]. The Frank-Starling law of the heart states that the stroke volume of the heart increases in response to an increase in the volume of blood filling the heart, i.e., the end diastolic volume, when all other factors remain constant, but this initial steep increase becomes progressively flat until a plateau is reached; in this last case, a further increase in preload does not lead to any significant increase in SV. According to this concept, the maneuver of fluid bolus infusion can be classified as responsive when it is followed by a significant increase of CO or SV [6]. The problem with this concept is that SV or CO is not a quantity that can be continuously measured. In fact, the available monitoring systems can provide a quantitative measure of cardiac output and stroke volume only at one time instant by the gold standard method of thermodilution, while the beat-to-beat variations of these quantities can be derived indirectly by other measurements such as arterial blood pressure.

Many indices have been proposed to predict FR, which are considered related to the Frank-Starling curve and a sort of an estimation of curve slope, i.e., the so-called dynamic indices (pulse pressure variation (PPV), stroke volume variation (SVV), systolic pressure variation (SPV), and others) [2]. However, these studies mainly concern ICU settings, septic, or postoperative patients $[7,8]$.

Very few studies have been performed in major surgery and in particular during liver surgery or OLT. Furthermore, these studies differ in terms of observational time window, type of fluid, velocity of administration, acquisition systems, and indices provided [5,9-12]. All these factors can explain the controversial results.

The originality of this work consists in the comparison between several approaches for the classification of the maneuvers and between indices provided by a commercial monitor and indices computed by algorithms described in literature. In fact, the majority of the works on FR indices take thresholds and criteria to identify responsive maneuver for granted [5,9-12]. The objective of this work is to improve the knowledge about the performance of these indices in association to different classification approaches in a particular setting, i.e., liver surgery. 
The specific aims are (1) the comparison of different $\mathrm{CO}$ estimators from invasive $\mathrm{ABP}$ measurement with particular attention to the assessment of $\mathrm{CO}$ variation after fluid administration and (2) the comparison of several indices for the prediction of fluid responsiveness of maneuvers classified from the $\mathrm{CO}$ measurements provided by a commercial monitor (PiCCO ${ }^{\mathrm{m}}$, Pulsion Medical Systems SE, Feldkirchen, Germany).

The next sections will present the experimental protocol and the approaches adopted for the classification of the maneuvers, the comparisons among the different $\mathrm{CO}$ estimators and the values obtained from a commercial monitor, the correlation between $\mathrm{CO}$ variations and FR predictor indices, and the comparisons between the FR indices of responsive and nonresponsive maneuvers.

\section{Methods}

\section{Study design and settings}

Ten patients, who fulfill inclusion and exclusion criteria, were enrolled in this observational study at the University Hospital Tor Vergata in Rome, Italy. The study was approved by the local Ethics Committee of University Hospital Tor Vergata, and the patients gave their written informed consent to participate.

\section{Data collection and protocol}

Inclusion criteria were age above 18 years and prescription of OLT or hepatectomy. The exclusion criteria were persistent arrhythmias, arteriosclerosis, and tidal volume less than $8 \mathrm{ml} / \mathrm{kg}$ of ideal weight [13]. OLTs were all performed by using piggyback technique, i.e., a venous anastomosis and not a venous-venous bypass.

Sedation was induced using propofol and/or sufentanil $(2 \mathrm{mg} / \mathrm{kg})$ and maintained by total intravenous anesthesia (TIVA, 6 to $8 \mathrm{mg} / \mathrm{kg} / \mathrm{h}$ ). Rapid infusions only were analyzed, and they consisted boluses of 100 or $500 \mathrm{ml}$ administered within $30 \mathrm{~s}$ or 1 min, respectively. The administered fluid consists mainly of blood recovered by the patient with addition of crystalloid and colloids; such solution is named 'reservoir'. The fluid was infused by a peristaltic pump (Belmont FMS 2000 MA, USA). Each patient was instrumented with an arterial catheter inserted in the brachial artery and placed in the aortic arch, with a central venous catheter inserted in a jugular vein and with an ECG lead. All the patients were monitored by Pulsion PiCCO and GE S/5 Avance Carestation devices.

\section{Measurements and preprocessing}

The following signals were continuously recorded during the entire surgery: arterial blood pressure $(\mathrm{ABP})$, air flow $(\mathrm{AF})$, air pressure (AP), central venous pressure (CVP), pulse contour cardiac output (PCCO), and SV. ABP and CVP were recorded at a sample frequency of $100 \mathrm{~Hz}, \mathrm{AF}$ and AP at $25 \mathrm{~Hz}$, and ECG at $300 \mathrm{~Hz}$. Cardiac output $(\mathrm{CO})$ and all indices provided by Pulsion PiCCO were provided with a sampling frequency of $2.5 \mathrm{~Hz}$.

For each maneuver, the time windows were selected beginning $20 \mathrm{~s}$ before the start of infusion and including the following 3 min. Preprocessing of raw recordings of ABP, ECG, CVP, and respiration (AF and AP) was performed in order to extract beat-by-beat series, employing standard and robust algorithms. In particular, $\mathrm{R}$ peaks indicative of 
each cardiac cycle were extracted through ECG processing, hence constructing RR interval series (RRI); beat-by-beat series of systolic blood pressure (SBP), diastolic blood pressure (DBP), mean arterial pressure (MAP), and pulse pressure (PP), computed as the difference between SBP of the current cardiac cycle and DBP of the previous cycle, were extracted from the arterial pressure waveform [14]; beat-by-beat CVP was calculated as the mean value of continuously recorded CVP over each cardiac cycle, defined as the interval between two consecutive $\mathrm{R}$ peaks. The respiratory cycles were identified from the AF signal by applying a threshold to a series obtained from the cross-correlation between the AF signal and a stepwise weight function. The tidal volume $V_{\mathrm{t}}$ was estimated as the area of AF signal between the beginning and the end of a respiratory cycle.

\section{Cardiac output estimators}

Two algorithms were implemented to extract beat-to-beat values of $\mathrm{CO}$ and SV from the continuous $\mathrm{ABP}$ signal: the Liljestrand and Zander method $\left(\mathrm{CO}_{\mathrm{LM}}, \mathrm{SV}_{\mathrm{LM}}\right)$ and the systolic area method $\left(\mathrm{CO}_{\mathrm{SA}}, \mathrm{SV}_{\mathrm{SA}}\right)$. For mathematical details, see [15].

As the $\mathrm{CO}$ and $\mathrm{SV}$ estimated on a beat-to-beat basis reflect the respiratory oscillations, a moving average filter was applied and no calibration was performed. In fact, the responsiveness of a maneuver is estimated as a percentage variation of $\mathrm{CO}$, and it was assessed as

$$
\Delta \mathrm{CO}(\%)=\frac{\mathrm{CO}-\mathrm{CO}_{\mathrm{ref}}}{\mathrm{CO}_{\mathrm{ref}}},
$$

where $\mathrm{CO}_{\text {ref }}$ is the average of $\mathrm{CO}$ values measured during the last $20 \mathrm{~s}$ before the start of infusion. $\mathrm{CO}$ variation was assessed by using the values obtained from the implemented algorithms and the values provided by PiCCO. The PiCCO is a commercial monitor that can provide both a quantitative measure of $\mathrm{CO}$ by means of thermodilution and a continuous estimation of $\mathrm{CO}$. The monitor estimates $\mathrm{CO}$ from the peripheral ABP measurement by applying the pulse contour analysis, i.e., a sort of weighted systolic area. Notice that $\mathrm{CO}$ variation is equal to cardiac index $(\mathrm{CI})$ variation as the normalizing term of body surface area is canceled.

\section{Fluid responsiveness indices}

PPV and SVV were estimated according to the definitions (2) and (3):

$$
\mathrm{PPV}(\%)=2 \frac{\mathrm{PP}_{\max }-\mathrm{PP}_{\min }}{\mathrm{PP}_{\max }+\mathrm{PP}_{\min }},
$$

where $\mathrm{PP}_{\max }$ and $\mathrm{PP}_{\text {min }}$ refer to the maximum and minimum values, respectively, obtained in a single respiratory cycle, previously identified by AF signal;

$$
\operatorname{SVV}(\%)=2 \frac{\mathrm{SV}_{\max }-\mathrm{SV}_{\text {min }}}{\mathrm{SV}_{\max }+\mathrm{SV}_{\text {min }}},
$$

where $\mathrm{SV}_{\max }$ and $\mathrm{SV}_{\text {min }}$ refer to the maximum and minimum values, respectively, obtained in a single respiratory cycle. The same indices provided by $\mathrm{PiCCO}$ monitor were recorded as well ( $\mathrm{PPV}_{\mathrm{PiCCO}}$ and $\mathrm{SVV} \mathrm{PiCCO}_{\mathrm{PiC}}$ ), for comparison purposes. Notice that the PiCCO monitor computed these indices without the information on respiratory cycle, but by considering moving windows of $30 \mathrm{~s}$. 
SPV was computed too as the difference between the maximum and minimum values of SBP obtained in a single respiratory cycle. The average values of these fluid responsiveness indices were computed in the time interval of $60 \mathrm{~s}$ before the fluid administration started.

\section{Criteria for classification of the maneuver}

Several approaches were adopted in order to classify the maneuvers into responsive and nonresponsive. Each maneuver was considered individually as the hemodynamic characteristics of a patient can be different at different stages of the surgical procedure and after short time intervals. The maneuvers were classified as responsive by using the values provided by the commercial monitor commonly adopted in the OR $\left(\mathrm{CO}_{\mathrm{PCCO}}\right)$ and according to different criteria, i.e., the time of response and the statistics for $\mathrm{CO}$ variation values. In particular,

- the $\mathrm{CO}$ variation, i.e., $\triangle \mathrm{CO}_{\mathrm{PCCO}}$, was assessed in two different time windows: after 1 min of fluid infusion and during the following 2 min;

- in each time window, the maximum value and the $75^{\circ}$ percentile of $\triangle \mathrm{CO}_{\mathrm{PCCO}}$ were estimated. The last case was considered to limit the effects of artifacts or short transitory increase in $\mathrm{CO}$, but not effective.

In all cases, a maneuver was classified as responsive if the $\mathrm{CO}$ variation estimated according to that approach was higher than $10 \%$.

\section{Statistical analysis}

The $\mathrm{CO}$ variations $(\triangle \mathrm{CO})$ obtained from different estimators and on different time intervals or statistics were compared, and a Pearson correlation analysis was performed. The fluid responsiveness indices estimated were compared with the FR indices provided by the commercial monitor PiCCO by the Bland-Altman analysis. After the subdivision of the maneuvers into two groups, i.e., responsive and not responsive, receiver operating characteristic (ROC) curves were estimated to obtain threshold values for each index. The index values were compared between the groups by means of Wilcoxon-Mann-Whitney test.

\section{Results}

Table 1 illustrates the characteristics of the population. Ten different surgical interventions were analyzed (eight hepatectomies and two OLTs), for a total of 25 maneuvers. The most frequent motivation of the surgery was neoplasia (seven out of ten).

\section{Comparisons of $\mathrm{CO}$ estimators}

Table 2 shows the correlation coefficients obtained by considering the $\mathrm{CO}$ variations estimated with the proposed methods and the $\mathrm{CO}$ variations calculated with the values provided by the monitor PiCCO. The higher significant coefficients were obtained for the second time window and for the Liljestrand and Zander method. Figure 1 shows the $\mathrm{CO}$ variation values for all the maneuvers considered in this study and for all the methods considered. Notice that the values are distributed mainly on the bisectrix, and 
Table 1 Patients' data

\begin{tabular}{lccccc}
\hline Subject & Age (years) & Sex (M/F) & Height $(\mathbf{c m})$ & Weight $(\mathbf{k g})$ & Type of surgery (H/OLT) \\
\hline$\# 1$ & 60 & F & 160 & 65 & $\mathrm{H}$ \\
$\# 2$ & 64 & F & 166 & 75 & $\mathrm{H}$ \\
$\# 3$ & 76 & M & 170 & 75 & $\mathrm{H}$ \\
$\# 4$ & 55 & M & 167 & 67 & $\mathrm{H}$ \\
$\# 5$ & 53 & M & 172 & 80 & $\mathrm{H}$ \\
$\# 6$ & 76 & F & 160 & 61 & $\mathrm{H}$ \\
$\# 7$ & 63 & F & 164 & 70 & OLT \\
$\# 8$ & 63 & M & 170 & 94 & $\mathrm{H}$ \\
$\# 9$ & 55 & $\mathrm{M}$ & 169 & 78 & $\mathrm{OLT}$ \\
$\# 10$ & 58 & $\mathrm{M}$ & 172 & 78 & $\mathrm{H}$ \\
& $62 \pm 8$ & $5 / 5$ & $167 \pm 4$ & $74 \pm 9$ & $8 / 2$ \\
\hline
\end{tabular}

$\mathrm{F}$, female; $\mathrm{M}$, male; $\mathrm{H}$, hepatectomy (hepatic resection); OLT, orthotopic liver transplantation.

the values are coherent with the ones from $\mathrm{PiCCO}$ as the values are in the same quadrant.

\section{Correlation between $\mathrm{CO}$ variation and fluid responsiveness indices}

The indices of fluid responsiveness were compared by the Bland-Altman analysis. As Figure 2 shows, the PPV values obtained from the monitor PiCCO showed a high agreement with the ones estimated according with the definition, i.e., by using the respiratory signal. The average value of the difference is close to zero, and the standard deviation of the difference is less than 5\%. A different result was obtained with SVV indices: $S V V_{S A}$ values were very similar to $S V V_{\mathrm{PiCCO}}$ for lower values, while for higher values, $\mathrm{SVV}_{\mathrm{SA}}$ overestimated the values of the monitor. $S V V_{\mathrm{LM}}$ showed higher differences with respect to $\mathrm{SVV}_{\mathrm{PiCCO}}$ and overestimated for low values; the average difference was $6.15 \%$.

The correlation coefficients between the fluid responsiveness indices and the $\mathrm{CO}$ variations estimated from the PiCCO values are illustrated in Table 3. The highest significant correlations were obtained by the PPV and SVV indices provided by the $\mathrm{PiCCO}$ monitor. Interestingly, none of the indices were correlated with the $\mathrm{CO}$ variation estimated in the first time window, i.e., during the first minute after infusion.

\section{Comparisons between responsive and non-responsive maneuvers}

Finally, the maneuvers were classified as responsive and non-responsive according the $\mathrm{CO}$ variation estimated as previously described. The $\mathrm{CO}$ variation estimated in

Table 2 Pearson correlation coefficients of $\mathrm{CO}$ variations

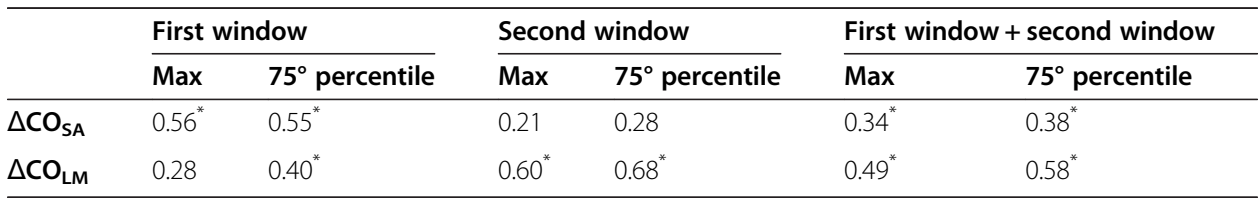

The coefficients were calculated between the values of $\mathrm{CO}$ variations estimated with the two methods and the variations estimated with the $\mathrm{CO}$ values provided by the monitor PiCCO. Fist window: 1 min after infusion; Second window: 2 minutes following the first window. *Pearson correlation $p$ value $<0.05$. 

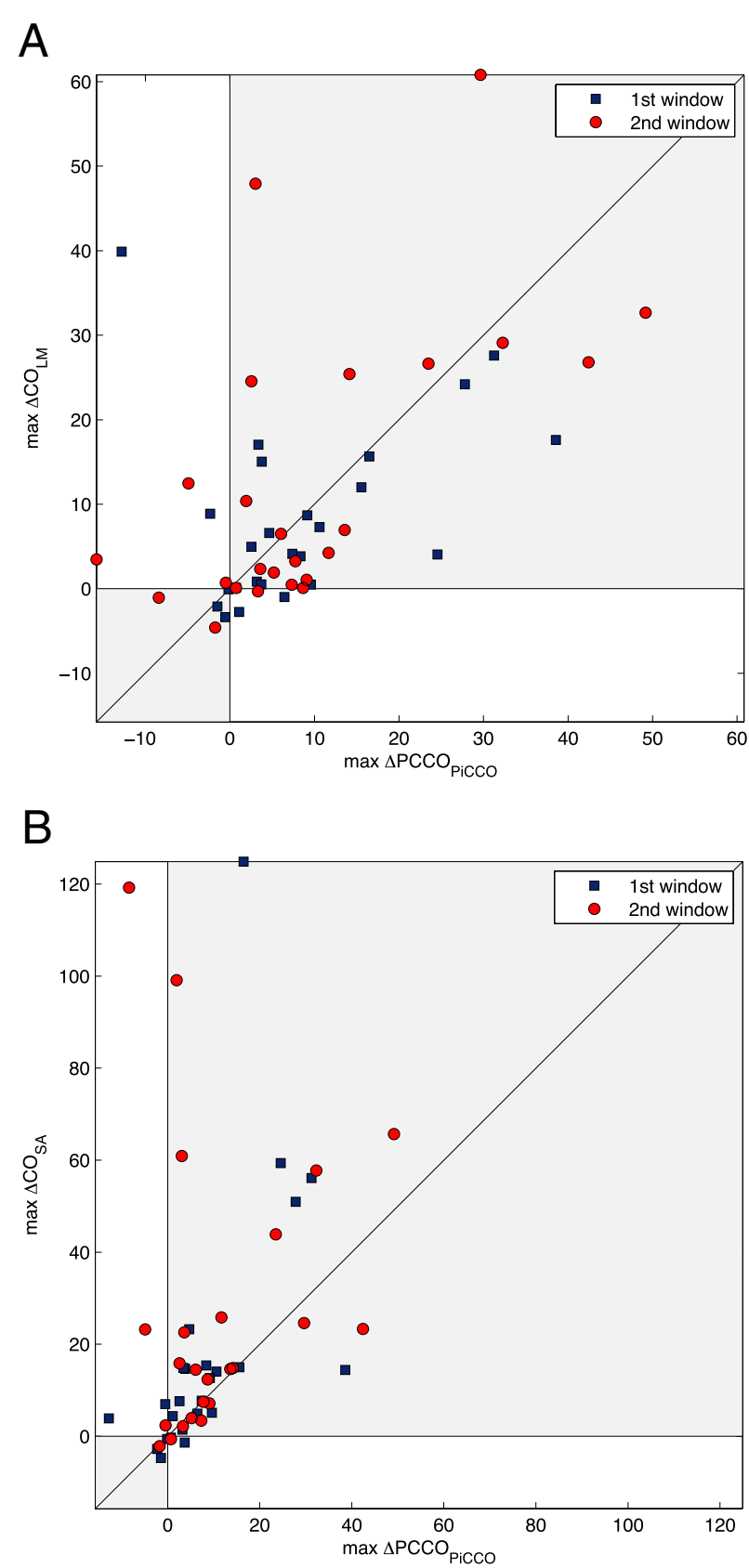

Figure 1 The figure shows the values of $\mathrm{CO}$ variations estimated with the two estimators. The figure shows the values of $\mathrm{CO}$ variations estimated with the two estimators (panel $\mathbf{A}$ : Liljestrand and Zander method; panel B: systolic area method).

the first time window, i.e., after the first minute following infusion, was excluded as none of the FR indices correlated to $\triangle \mathrm{PCCO}$. The maneuvers classified according to the maximum values of $\triangle \mathrm{PCCO}$ estimated during the second time window obtained the best performance in terms of sensitivity and specificity. Table 4 shows the area under curve (AUC) and the threshold values obtained from the ROC curve. In

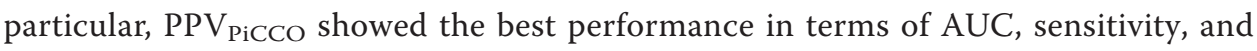
specificity. 


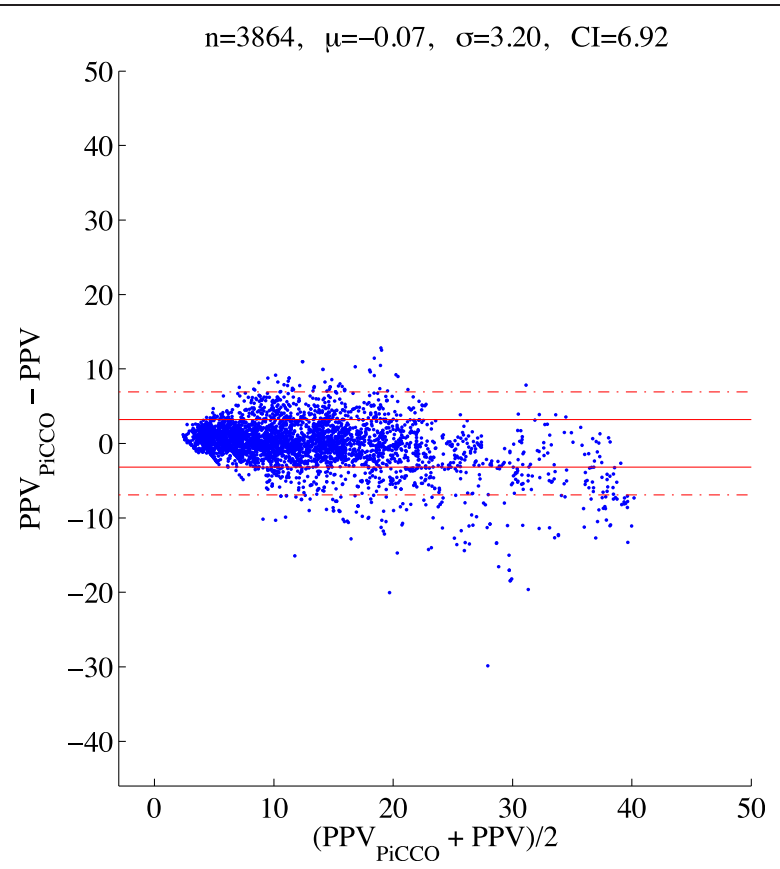

Figure 2 The figure shows the values from Bland-Altman analysis of the PiCCO. The figure shows the values from Bland-Altman analysis of the PPV values provided by PiCCO.

However, the PPV threshold (14\%) values were slightly higher than the values reported in the literature. A larger difference was instead obtained for the SVV index whose threshold was twice the ones reported in the literature and very different among the SVV estimators considered in this study (see Table 4 and Figure 3).

\section{Discussion}

This work showed how $\mathrm{CO}$ estimated from invasive $\mathrm{ABP}$ measurement by different algorithms provided similar performances for the assessment of $\mathrm{CO}$ variation after fluid administration. This analysis was not focused on the validation of $\mathrm{CO}$ absolute values obtained from the application of different algorithms existing in the literature, but rather to verify the possibility to use such standard methods, in the absence of

Table 3 Pearson correlation coefficients between CO variations and FR indices

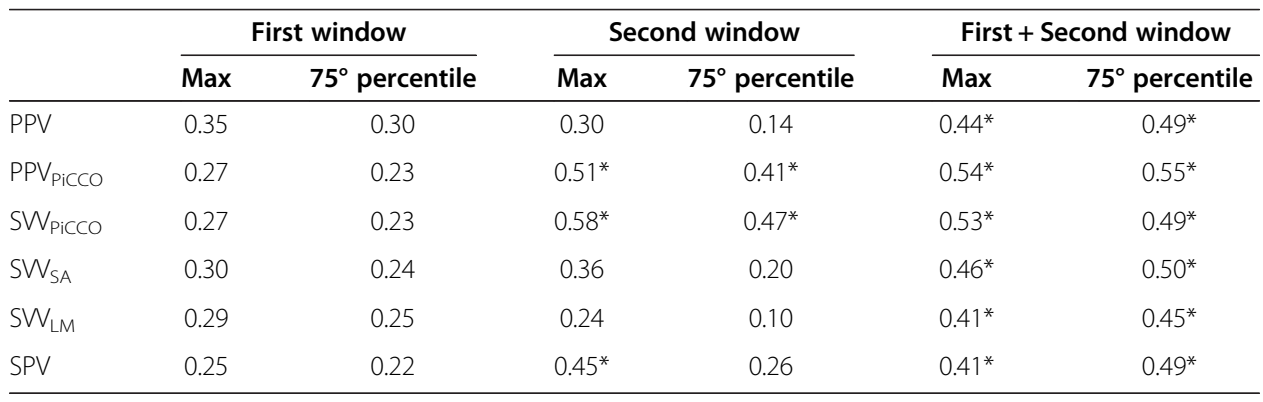

The coefficients were calculated between the FR indices values estimated before fluid infusion and the variation computed from the $\mathrm{CO}$ values provided by the monitor PiCCO after the infusion in three different time windows. $1^{\text {st }}$ window: one minute after infusion; $2^{\text {nd }}$ window: two minutes following the first window. * Pearson correlation $p$-value $<0.05$. 
Table 4 Values from the ROC curve of the FR indices

\begin{tabular}{lcccc}
\hline & Threshold value & Sensitivity & Specificity & AUC \\
\hline PPV & 13.9 & 0.88 & 0.86 & 0.82 \\
PPV $_{\text {PiCCO }}$ & 14.4 & 0.88 & 0.86 & 0.92 \\
SW $_{\text {PiCCO }}$ & 19.7 & 0.88 & 0.75 & 0.88 \\
SW $_{\text {SA }}$ & 18.2 & 0.82 & 0.71 & 0.80 \\
SW & 9.4 & 0.76 & 0.71 & 0.74 \\
SPV & 8.1 & 0.88 & 0.86 & 0.87 \\
\hline
\end{tabular}

Threshold, sensitivity, specificity, and AUC values are estimated from the ROC curve. These values refer to the maneuvers classified according to the maximum value of $\mathrm{CO}$ variation registered in the second time window.

thermodilution calibration. Also, the estimates of cardiac output provided by commercial monitor systems are not continuous and often, but not always, require calibration; they are ultimately determined by algorithms that are covered by patents, making it hard to evaluate the goodness with respect to other analyses, such as the ones performed offline in this work.

As regards the comparison among the indices for the prediction of fluid responsiveness, the Bland-Altman analysis showed a good agreement between the PPV values estimated according to the definition, i.e., by using the respiratory signal, and the PPV values provided by the monitor PiCCO $^{\text {ma }}$ (Pulsion). The SVV indices showed instead a

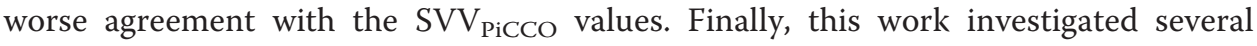
approaches in order to classify the maneuvers into responsive and nonresponsive to evaluate their performance in liver surgery. The maneuvers were classified as responsive by using the values provided by the commercial monitor $\left(\mathrm{PiCCO}^{\mathrm{m}}\right.$, Pulsion) commonly adopted in operating rooms $\left(\mathrm{CO}_{\mathrm{PCCO}}\right)$ and by adopting different criteria, i.e., the time of response and the statistics for $\mathrm{CO}$ variation values. $\mathrm{PPV}_{\mathrm{PiCCO}}$ showed the best performance in terms of AUC, sensitivity, and specificity when the maneuvers were classified according

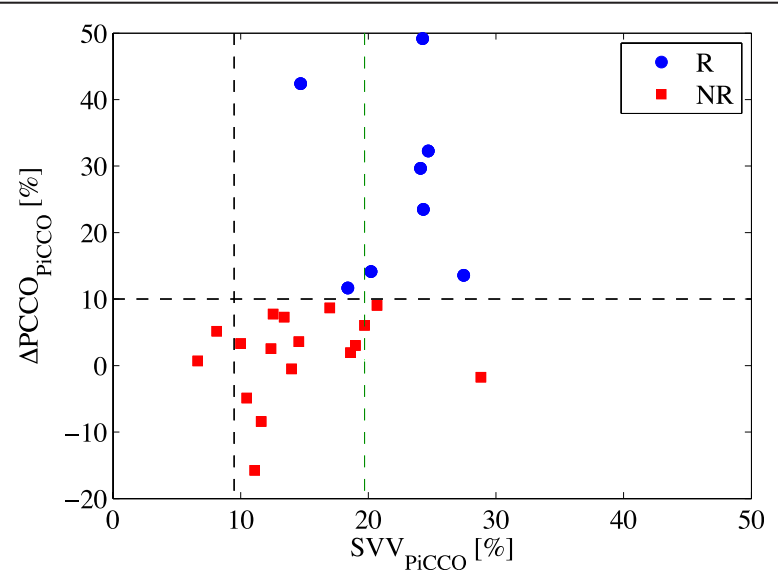

Figure 3 Values of SVV indices and CO variations. Values of SW estimated by PiCCO before the rapid infusion and values of maximum variation of cardiac output ( $\triangle \mathrm{PCCO}$ ) obtained during the second time window. The dashed horizontal line marks the threshold, which separates the responsive (R) from nonresponsive (NR) maneuvers. The dashed black vertical line marks the threshold commonly used in the literature (9.5\%) for SW index in order to predict the outcome [15]. The green line marks the threshold obtained in the present work. 
to the criterion of maximum $\mathrm{CO}$ variation during the 2 minutes following 1 minute after fluid infusion. Moreover, the threshold value obtained from the ROC curve was $14 \%$ similar to those reported in the literature on ICU setting for septic patients (13\%) [16] or cardiac surgery (12\%) [17]. SVV indices showed good performance too; however, the threshold values were different among the estimators (Table 4) and much higher than those reported in literature [18].

In the work of Biais et al. [12], the FR maneuvers were analyzed in patients undergoing OLT, and the SVV threshold values observed in association with the best classification performance were $9 \%$ for the SVV index estimated by a Doppler echography and $10 \%$ for the SVV index estimated by FlocTrac (Edward Lifescience, Irvine, CA). However, such differences can be explained by the different protocol: it has not been specified the time interval before fluid infusion and the volume expansion maneuver took $20 \mathrm{~min}$.

Our results suggested that PPV indices are more reliable and computationally feasible than SVV indices. This can be easily explained by the fact that the former ones are estimated from a direct measure, i.e., from ABP signal; the latter ones are assessed in turn from an estimated quantity, i.e., the stroke volume assessed from ABP signal. This further exposes the index to the potential limitations, which should be carefully considered when relying on estimates from monitors.

\section{Conclusions}

The value of this work consists in the approaches proposed for the classification of the maneuvers and the thorough analyses of the FR indices both provided by a commercial monitor and computed by algorithms well known in the literature. This solution represents a novelty and can pave the way for further analyses in order to standardize and validate the criteria proposed. Moreover, the commercial monitor provides only the values of the indices, and the variations are often visually observed by the clinicians. This could be a source of errors or, at the very least, a limitation of the effectiveness of monitor-derived estimates. The offline investigation represents a limitation, but it is the first step for the implementation of new monitoring procedures, e.g., monitoring systems for the fluid management, which can control the fluid infusion and monitor the patient response automatically [19].

The main finding of this work is that PPV indices are more reliable and computationally feasible than SVV indices, and PPV AUC, sensitivity, and specificity when the maneuvers were classified according to the maximum values of $\triangle \mathrm{PCCO}$ estimated during the second time window. The threshold values produced by the PPV indices were similar between the ones provided by the commercial monitor and the computed index, in further agreement with the literature. From these observations, PPV index seems more suitable for FR prediction in liver surgery.

\footnotetext{
Abbreviations

ABP: Arterial blood pressure; AF: Air flow; AP: Air pressure; AUC: Area under curve; CO: Cardiac output; CVP: Central venous pressure; FR: Fluid responsiveness; PCCO: Pulse contour cardiac output; PP: Pulse pressure; PVV: Pulse pressure variation; ROC: Receiver operating characteristic; SPV: Systolic pressure variation; SV: Stroke volume; SVV: Stroke volume variation.
} 


\section{Authors' contributions}

$F C, M D$, and NT initiated and designed the study. AC, FC, and MD collected the data. MF, SP, FA, and GB analyzed and interpreted the data. MF, FA, SP, and GB contributed to the writing of the manuscript. $A C, F C, M G, M D$, and GB gave scientific advice in their field of expertise. All authors revised the manuscript and approved and contributed to its improvement.

\section{Acknowledgements}

This work was supported by the Italian Ministry of Education, University and Research (MIUR, FIRB2008, Project\# RBFR08VABD). We would like to thank the personnel of the Policlinico "Tor Vergata" for their commitment and dedication to the successful conduction of this study.

\section{Author details}

Department of Electronics, Information and Bioengineering (DEIB), Politecnico di Milano, Piazza Leonardo da Vinci 32, Milan 20133, Italy. ${ }^{2}$ Medical Physics Section, University of Rome Tor Vergata, Via Montpellier 1, Rome 00133 , Italy. ${ }^{3}$ Department of Anesthesia and Intensive Care Medicine, University of Rome Tor Vergata, Via Montpellier 1, Rome 00133, Italy.

Received: 11 August 2013 Accepted: 18 November 2013

Published: 10 January 2014

\section{References}

1. Vincent J-L, Gerlach H: Fluid resuscitation in severe sepsis and septic shock: an evidence-based review. Crit Care Med 2004, 32(11 Suppl):S451-S454.

2. Michard F, Teboul JT: Predicting fluid responsiveness in ICU patients: a critical analysis of the evidence. Chest 2002, 121:2000-2008.

3. Vincent J-L, Weil MH. Fluid challenge revisited. Crit Care Med 2006, 34(5):1333-1337.

4. Pinsky M: Functional hemodynamic monitoring. Intensive Care Med 2002, 28:386-388.

5. Gouvea G, Diaz R, Auler L, Toledo R, Martinho JM: Evaluation of the pulse pressure variation index as a predictor of fluid responsiveness during orthotopic liver transplantation. Br J Anaesth 2009, 103(2):238-243.

6. Michard F, Lopes MR, Auler JO Jr: Pulse pressure variation: beyond the fluid management of patients with shock. Crit Care 2007, 11(3):131.

7. Bendjelid K, Romand JA: Fluid responsiveness in mechanically ventilated patients: a review of indices used in intensive care. Intensive Care Med 2003, 29(3):352-360.

8. Marik PE, Baram M: Noninvasive hemodynamic monitoring in the intensive care unit. Crit Care Clin 2007, 23(3):383-400

9. Su BC, Tsai YF, Cheng CW, Yu HP, Yang MW, Lee WC, Lin CC: Stroke volume variation derived by arterial pulse contour analysis is a good indicator for preload estimation during liver transplantation. Transplant Proc 2012, 44(2):429-432.

10. Shin YH, Ko JS, Gwak MS, Kim GS, Lee JH, Lee SK: Utility of uncalibrated femoral stroke volume variation as a predictor of fluid responsiveness during the anhepatic phase of liver transplantation. Liver Transp/ 2011, 17(1):53-59.

11. Kim YK, Shin WJ, Song JG, Jun IG, Kim HY, Seong SH, Hwang GS: Comparison of stroke volume variations derived from radial and femoral arterial pressure waveforms during liver transplantation. Transplant Proc 2009, 41(10):4220-4228.

12. Biais M, Nouette-Gaulain $K$, Roullet $S$, Quinart A, Revel P, Sztark F: A comparison of stroke volume variation measured by Vigileo/FloTrac system and aortic Doppler echocardiography. Anesth Analg 2009, 109(2):466-469.

13. Kim HK, Pinsky MR: Effect of tidal volume, sampling duration, and cardiac contractility on pulse pressure and stroke volume variation during positive-pressure ventilation. Critical Care Med 2008, 36(10):2858-2862.

14. Zong W, Heldt T, Moody GB, Mark RG: An open-source algorithm to detect onset of arterial blood pressure pulses. In CinC2003. Comput Cardiol 2003, 30:259-262.

15. Sun JX, Reisner AT, Saeed M, Heldt T, Mark RG: The cardiac output from blood pressure algorithms trial. Crit Care Med 2009, 37(1):72-80

16. Lamia B, Chemla D, Richard C, Teboul JL: Clinical review: interpretation of arterial pressure wave in shock states. Crit Care 2005, 9(6):601-606.

17. Auler JO Jr, Galas F, Hajjar L, Santos L, Carvalho T, Michard F: Online monitoring of pulse pressure variation to guide fluid therapy after cardiac surgery. Anesth Analg 2008, 106(4):1201-1206.

18. Berkenstadt H, Margalit N, Hadani M, Friedman Z, Segal E, Villa Y, Perel A: Stroke volume variation as a predictor of fluid responsiveness in patients undergoing brain surgery. Anesth Analg 2001, 92(4):984-989.

19. Rinehart J, Liu N, Alexander B, Cannesson M: Review article: closed-loop systems in anesthesia: is there a potential for closed-loop fluid management and hemodynamic optimization? Anesth Analg 2012, 114(1):130-143.

doi:10.1186/2194-3990-1-6

Cite this article as: Ferrario et al: Fluid responsiveness in liver surgery: comparisons of different indices and approaches. Journal of Computational Surgery 2014 1:6. 